\title{
A fully nonlinear problem arising in financial modelling
}

\author{
Maria do Rosário Grossinho ${ }^{* *}$ and Eva Morais ${ }^{1,2}$
}

\author{
"Correspondence: mrg@iseg.utl.pt \\ ${ }^{1}$ ISEG, CEMAPRE - Technical \\ University of Lisbon, Rua do \\ Quelhas 6, Lisboa, 1200-781, \\ Portugal \\ Full list of author information is \\ available at the end of the article
}

\begin{abstract}
We state existence and localisation results for a fully nonlinear boundary value problem using the upper and lower solutions method. With this study we aim to contribute to a better understanding of some analytical features of a problem arising in financial modelling related to the introduction of transaction costs in the classical Black-Scholes model. Our result concerns stationary solutions which become interesting in finance when the time does not play a relevant role such as, for instance, in perpetual options.
\end{abstract}

\section{Introduction}

In 1973, Fisher Black and Myron Scholes suggested a model that became fundamental for the valuation of financial derivatives in a complete frictionless market. Along with the noarbitrage possibilities, the classical Black-Scholes model assumes that in order to replicate exactly the returns of a certain derivative, the hedging portfolio is continuously adjusted by transactioning the underlying asset of the derivative. This fact can only happen if no transaction costs exist when buying or selling financial assets. Otherwise, a continuous adjustment would imply that those costs, such as taxes or fees, would become infinitely large.

Hence the introduction of transaction costs in the model is a problem that has been motivating the work of several authors and has led to the study of new models that generalise the classical Black-Scholes model.

In this paper, we aim to give a contribution to better understanding of some analytical features of the problem. We are concerned with the existence and localisation results for the nonlinear second-order Dirichlet boundary problem

$$
\left\{\begin{array}{l}
\left.x^{3}\left(V^{\prime \prime}\right)^{2}+p x^{2} V^{\prime \prime}+q x V^{\prime}=q V \quad \text { in }\right] c, d[ \\
V(c)=V_{c}, \quad V(d)=V_{d},
\end{array}\right.
$$

where $0<c<d$ and $p, q$ are positive constants ( $p$ and $q$ can be assumed nonnegative, but the assumption is neither interesting from the mathematical viewpoint nor reasonable for applications to finance). We also consider that $V_{c} \leq V_{d}$. This assumption turns out to be quite natural in some financial settings, for instance, if we are dealing with call options.

This problem is related to the study of stationary solutions of a nonlinear parabolic equation that models the valuation of a call option in presence of transaction costs. These stationary solutions give the option value $V$ as a function of the stock price, which can be

(c) 2013 Grossinho and Morais; licensee Springer. This is an Open Access article distributed under the terms of the Creative Commons Attribution License (http://creativecommons.org/licenses/by/2.0), which permits unrestricted use, distribution, and reproduction in any medium, provided the original work is properly cited. 
interesting when dealing with a model where the time does not play a relevant role such as, for instance, in perpetual options.

Since $x \in[c, d]$ with $c>0$, the equation of (1) is of the following type:

$$
\left(V^{\prime \prime}\right)^{2}+v(x) V^{\prime \prime}+\psi(x) V^{\prime}=\mu(x) V
$$

However, we will consider it in the form presented in (1) in order to keep some similarity to the financial setting problem, as we will see later.

The arguments used rely on the upper and lower solutions method (see [1, 2]).

The results we present were motivated by the work of Amster et al. (see [3]). There, the authors studied an analogous problem but without obtaining a localisation result. Then they proceeded their study using a limit of a nonincreasing (nondecreasing) sequence of upper (respectively lower) solutions. Those results were proved under some Lipschitz condition, which has some financial implications. We do not assume such a condition and, moreover, we obtain localisation information on the solution.

In this paper, we state some existence results for the problem (1), following a previous study contained in [4], and improving the localisation result. Relating to the financial model, this implies more accuracy in the information of the option regarding the behaviour of the stock value, in a model where transaction costs are considered. In Section 2, we consider an auxiliary problem. Using the results proved there, we state in Section 3 an existence and localisation theorem for (1). In Section 4, we make some comments on the financial problem that suggested our analytical study. With this section we aim to illustrate the interplay between finance and mathematics which, in fact, is very challenging and often stimulates the use of innovative mathematical and computational techniques.

\section{Auxiliary result}

Observe that the equation of (1) is from the algebraic point of view a second-order equation in the variable $Y=V^{\prime \prime}$. So, solving it algebraically in order of $V^{\prime \prime}$, we obtain the equivalent form

$$
V^{\prime \prime}=\frac{-p x^{2} \pm \sqrt{p^{2} x^{4}-4 q x^{3}\left(x V^{\prime}-V\right)}}{2 x^{3}}
$$

which leads us to consider the equation

$$
V^{\prime \prime}+H_{ \pm}\left(x, V, V^{\prime}\right)=0
$$

where

$$
H_{ \pm}\left(x, V, V^{\prime}\right)=\frac{p x^{2} \mp \sqrt{p^{2} x^{4}-4 q x^{3}\left(x V^{\prime}-V\right)}}{2 x^{3}} .
$$

This fact suggests the study of the auxiliary problem

$$
\left\{\begin{array}{l}
V^{\prime \prime}+H\left(x, V, V^{\prime}\right)=0, \\
V(c)=V_{c}, \quad V(d)=V_{d},
\end{array}\right.
$$


where

$$
H\left(x, V, V^{\prime}\right)=\frac{p x^{2}-\sqrt{p^{2} x^{4}+4 q x^{3}\left|x V^{\prime}-V\right|}}{2 x^{3}} .
$$

The main argument to prove Theorem 2 relies on the method of upper and lower solutions.

We recall that $\alpha \in C^{2}$ is a lower solution of (2) if

$$
\left\{\begin{array}{l}
\alpha^{\prime \prime}+H\left(x, \alpha, \alpha^{\prime}\right) \geq 0, \\
\alpha(c) \leq V_{c}, \quad \alpha(d) \leq V_{d}
\end{array}\right.
$$

Similarly, an upper solution $\beta \in C^{2}$ of (2) is defined by reversing the inequalities in (3). A solution of (2) is a function $u$ which is simultaneously a lower and an upper solution. A function $f$ is said to satisfy the Nagumo condition on some given subset $E \subset I \times \mathbb{R}^{2}$ if there exists a positive continuous function $\varphi \in C\left(\mathbb{R}_{0}^{+},[\varepsilon,+\infty[), \varepsilon>0\right.$, such that

$$
|f(x, y, z)| \leq \varphi(|z|), \quad \forall(x, y, z) \in E,
$$

and

$$
\int_{0}^{+\infty} \frac{s}{\varphi(s)} d s=+\infty
$$

Lemma 1 Suppose that

$$
\frac{V_{d}}{d} \leq \frac{V_{c}}{c}
$$

Then:

1. The function $\alpha_{1}(x)=\frac{V_{d}}{d} x$ is a lower solution of the problem (2).

2. If $k=\sqrt{\frac{q}{c^{3}}}$ is small enough, then the function

$$
\alpha_{2}(x)=\frac{V_{d}-V_{c}}{d^{2}-c^{2}} x^{2}+\frac{d^{2} V_{c}-c^{2} V_{d}}{d^{2}-c^{2}}
$$

is a lower solution of the problem (2).

3. The function

$$
\beta(x)=\frac{V_{d}-V_{c}}{d-c} x+\frac{d V_{c}-c V_{d}}{d-c}
$$

is an upper solution of the problem (2).

Proof

1. If we plug $\alpha_{1}(x)=\frac{V_{d}}{d} x$ in the first member of the equation in (2), we have that

$$
\left(\frac{V_{d}}{d} x\right)^{\prime \prime}+H\left(x, \frac{V_{d}}{d} x,\left(\frac{V_{d}}{d} x\right)^{\prime}\right)=0+\frac{p x^{2}-\sqrt{p^{2} x^{4}-4 q x^{3}\left(\frac{V_{d}}{d} x-\frac{V_{d}}{d} x\right)}}{2 x^{3}}=0
$$


and by (5)

$$
\alpha_{1}(c)=\frac{V_{d}}{d} c \leq V_{c}, \quad \alpha_{1}(d)=\frac{V_{d}}{d} d=V_{d} .
$$

2. Consider now the function

$$
\alpha_{2}(x)=\frac{V_{d}-V_{c}}{d^{2}-c^{2}} x^{2}+\frac{d^{2} V_{c}-c^{2} V_{d}}{d^{2}-c^{2}} .
$$

Observe first that

$$
\alpha_{2}(c)=\frac{V_{d}-V_{c}}{d^{2}-c^{2}} c^{2}+\frac{d^{2} V_{c}-c^{2} V_{d}}{d^{2}-c^{2}}=V_{c}
$$

and

$$
\alpha_{2}(d)=\frac{V_{d}-V_{c}}{d^{2}-c^{2}} d^{2}+\frac{d^{2} V_{c}-c^{2} V_{d}}{d^{2}-c^{2}}=V_{d} .
$$

On the other hand, we have that

$$
\begin{aligned}
H\left(x, V, V^{\prime}\right) & =\frac{p x^{2}-\sqrt{p^{2} x^{4}+4 q x^{3}\left|x V^{\prime}-V\right|}}{2 x^{3}} \\
& \geq \frac{p x^{2}-\sqrt{p^{2} x^{4}}-\sqrt{4 q x^{3}\left|x V^{\prime}-V\right|}}{2 x^{3}} \\
& =-\sqrt{\frac{q}{x^{3}}} \sqrt{\left|x V^{\prime}-V\right|} \\
& \geq-\sqrt{\frac{q}{c^{3}}} \sqrt{\left|x V^{\prime}-V\right|} \\
& =-k \sqrt{\left|x V^{\prime}-V\right|},
\end{aligned}
$$

where $k=\sqrt{\frac{q}{c^{3}}}$.

So, if we plug the function $\alpha_{2}(x)$ in the first member of the equation in (2), we obtain

$$
\begin{aligned}
& \left(\frac{V_{d}-V_{c}}{d^{2}-c^{2}} x^{2}+\frac{d^{2} V_{c}-c^{2} V_{d}}{d^{2}-c^{2}}\right)^{\prime \prime} \\
& \quad+H\left(x, \frac{V_{d}-V_{c}}{d^{2}-c^{2}} x^{2}+\frac{d^{2} V_{c}-c^{2} V_{d}}{d^{2}-c^{2}},\left(\frac{V_{d}-V_{c}}{d^{2}-c^{2}} x^{2}+\frac{d^{2} V_{c}-c^{2} V_{d}}{d^{2}-c^{2}}\right)\right)^{\prime} \\
& \geq 2 \frac{V_{d}-V_{c}}{d^{2}-c^{2}}-k \sqrt{\left|2 x^{2} \frac{V_{d}-V_{c}}{d^{2}-c^{2}}-\frac{V_{d}-V_{c}}{d^{2}-c^{2}} x^{2}-\frac{d^{2} V_{c}-c^{2} V_{d}}{d^{2}-c^{2}}\right|} \\
& =2 \frac{V_{d}-V_{c}}{d^{2}-c^{2}}-k \sqrt{\left|x^{2} \frac{V_{d}-V_{c}}{d^{2}-c^{2}}-\frac{d^{2} V_{c}-c^{2} V_{d}}{d^{2}-c^{2}}\right|},
\end{aligned}
$$

which, for $k$ small enough, is non-negative.

3. Consider now the function

$$
\beta(x)=\frac{V_{d}-V_{c}}{d-c} x+\frac{d V_{c}-c V_{d}}{d-c} .
$$


Then, in an analogous way, we plug $\beta(x)$ in the first member of the equation in (2). We observe that

$$
\begin{aligned}
& \left(\frac{V_{d}-V_{c}}{d-c} x+\frac{d V_{c}-c V_{d}}{d-c}\right)^{\prime \prime} \\
& \quad+H\left(x, \frac{V_{d}-V_{c}}{d-c} x+\frac{d V_{c}-c V_{d}}{d-c},\left(\frac{V_{d}-V_{c}}{d-c} x+\frac{d V_{c}-c V_{d}}{d-c}\right)^{\prime}\right) \\
& =0+\frac{p x^{2}-\sqrt{p^{2} x^{4}+4 q x^{3}\left|\frac{V_{d}-V_{c}}{d-c} x-\frac{V_{d}-V_{c}}{d-c} x+\frac{d V_{c}-c V_{d}}{d-c}\right|}}{2 x^{3}}<0
\end{aligned}
$$

and

$$
\beta(c)=V_{c}, \quad \beta(d)=V_{d} .
$$

So, the three assertions of the lemma are proved.

Remark 1 The value $K^{*}=\frac{2\left(V_{d}-V_{c}\right)}{\left(d^{2}-c^{2}\right) \sqrt{V_{c}+V_{d}}}$ is a suitable upper bound for the possible values that $k=\sqrt{\frac{q}{c^{3}}}$ can take in the above assertion 2. In fact, for $x \in[c, d]$ and since $\frac{V_{d}}{d} \leq \frac{V_{c}}{c}$, easy computations show that

$$
\left|x^{2}\left(V_{d}-V_{c}\right)-\left(d^{2} V_{c}-c^{2} V_{d}\right)\right| \leq\left(d^{2}-c^{2}\right)\left(V_{d}+V_{c}\right)
$$

Therefore, if $k \leq K^{*}$, we have

$$
\begin{aligned}
k & \leq \frac{2\left(V_{d}-V_{c}\right)}{\left(d^{2}-c^{2}\right) \sqrt{V_{c}+V_{d}}} \\
& \leq \frac{2 \frac{V_{d}-V_{c}}{d^{2}-c^{2}}}{\sqrt{\left|x^{2} \frac{V_{d}-V_{c}}{d^{2}-c^{2}}-\frac{d^{2} V_{c}-c^{2} V_{d}}{d^{2}-c^{2}}\right|}} \text { for all } x \in[c, d],
\end{aligned}
$$

which is used in the last step of the proof of assertion 2.

Now we state an existence and localisation result for the auxiliary problem (2).

Theorem 1 Suppose that $\frac{V_{d}}{d} \leq \frac{V_{c}}{c}$. Then:

1. The problem (2) has a solution $V$ such that

$$
\frac{V_{d}}{d} x \leq V(x) \leq \frac{V_{d}-V_{c}}{d-c} x+\frac{d V_{c}-c V_{d}}{d-c} .
$$

2. If $k=\sqrt{\frac{q}{c^{3}}}$ is small enough, the problem (2) has a solution $V$ such that

$$
\frac{V_{d}-V_{c}}{d^{2}-c^{2}} x^{2}+\frac{d^{2} V_{c}-c^{2} V_{d}}{d^{2}-c^{2}} \leq V(x) \leq \frac{V_{d}-V_{c}}{d-c} x+\frac{d V_{c}-c V_{d}}{d-c} .
$$

3. If $\frac{V_{d}}{d}=\frac{V_{c}}{c}$, the function $V(x)=\frac{V_{d}}{d} x$ is a solution of the problem (2). 
Proof By the previous lemma, we know already that there are lower and upper solutions for the problem (2). It is also clear that they are well ordered, that is,

$$
\alpha_{1} \leq \beta \quad \text { and } \quad \alpha_{2} \leq \beta
$$

So, if the function $H$ satisfies the Nagumo condition, the thesis will follow by [1] or [2]. In order to prove assertions 1 and 2, we consider the sets, respectively,

$$
E_{1}=\left\{(x, y, z) \in[c, d] \times \mathbb{R}^{2}: \frac{V_{d}}{d} x \leq y \leq \frac{V_{d}-V_{c}}{d-c} x+\frac{d V_{c}-c V_{d}}{d-c}\right\}
$$

and

$$
E_{2}=\left\{(x, y, z) \in[c, d] \times \mathbb{R}^{2}: \frac{V_{d}-V_{c}}{d^{2}-c^{2}} x^{2}+\frac{d^{2} V_{c}-c^{2} V_{d}}{d^{2}-c^{2}} \leq y \leq \frac{V_{d}-V_{c}}{d-c} x+\frac{d V_{c}-c V_{d}}{d-c}\right\} .
$$

Function $H$ satisfies the Nagumo condition in $E_{1}$ and in $E_{2}$. In fact, we have that

$$
\begin{aligned}
|H(x, y, z)| & =\left|\frac{p x^{2}-\sqrt{p^{2} x^{4}+4 q x^{3}|x z-y|}}{2 x^{3}}\right| \\
& \leq \frac{p x^{2}+\sqrt{4 q x^{3}|x z-y|}}{2 x^{3}} \\
& \leq \frac{p}{2 x}+\sqrt{\frac{q}{x^{3}}} \sqrt{\frac{V_{d}-V_{c}}{d-c} x+\frac{d V_{c}-c V_{d}}{d-c}}+\frac{\sqrt{q}}{x} \sqrt{|z|} \\
& \leq \frac{p}{2 c}+\sqrt{\frac{q}{c^{3}}} \sqrt{\frac{V_{d}-V_{c}}{d-c} d+\frac{d V_{c}-c V_{d}}{d-c}}+\frac{\sqrt{q}}{c} \sqrt{|z|} .
\end{aligned}
$$

Thus, for some positive constants $k_{1}$ and $k_{2}$, we have that

$$
|H(x, y, z)| \leq k_{1}+k_{2} \sqrt{|z|}, \quad \forall(x, y, z) \in E_{1} \cup E_{2}
$$

and

$$
\int_{0}^{+\infty} \frac{s}{k_{1}+k_{2} \sqrt{|s|}} d s=+\infty
$$

So, by what was said above, the first two assertions of the thesis hold.

The third assertion follows directly from obvious computations.

Proposition 1 Consider the problem (2) and the solution $V$ given by Theorem 1 . Then $V$ is convex and satisfies $V(x) \geq x V^{\prime}(x)$ in $[c, d]$.

Proof The convexity of the solution $V$ follows easily by the equation of the problem (2)

$$
V^{\prime \prime}=-H\left(x, V, V^{\prime}\right)=\frac{-p x^{2}+\sqrt{p x^{4}+4 q x^{3}\left|x V^{\prime}-V\right|}}{2 x^{3}} \geq 0 .
$$

Noting that

$$
\left(x V^{\prime}(x)-V(x)\right)^{\prime}=x V^{\prime \prime}(x) \geq 0
$$


then

$$
x V^{\prime}(x)-V(x) \leq d V^{\prime}(d)-V_{d}
$$

But, using Theorem 1, assertion 1, we have that $V^{\prime}(d) \leq \frac{V_{d}}{d}$. In fact, since $\frac{V_{d}}{d} x \leq V(x)$, it follows that $\frac{V_{d}}{d} \geq \frac{V(x)-V_{d}}{x-d}$, for $c \leq x<d$. Letting $x \rightarrow d$, we obtain that $\frac{V_{d}}{d} \geq V^{\prime}(d)$.

Then we have

$$
x V^{\prime}(x)-V(x) \leq 0 .
$$

\section{Existence and localisation result}

We return to the original problem (1) and state an existence and uniqueness result. We also provide information on the localisation of the solution.

Theorem 2 Consider the nonlinear Dirichlet boundary value problem (1). The following assertions hold:

1. The function $V(x)=\frac{V_{c}}{c} x$ is a solution of the problem (1) if and only if $\frac{V_{d}}{d}=\frac{V_{c}}{c}$.

2. If $\frac{V_{d}}{d}<\frac{V_{c}}{c}$, then the problem (1) has a convex solution $V$ such that

$$
\frac{V_{d}}{d} x \leq V(x) \leq \frac{V_{d}-V_{c}}{d-c} x+\frac{d V_{c}-c V_{d}}{d-c} .
$$

3. If $\frac{V_{d}}{d}<\frac{V_{c}}{c}$ and $k=\sqrt{\frac{q}{c^{3}}}$ is small enough, then the problem (1) has a convex solution $V$ such that

$$
\frac{V_{d}-V_{c}}{d^{2}-c^{2}} x^{2}+\frac{d^{2} V_{c}-c^{2} V_{d}}{d^{2}-c^{2}} \leq V(x) \leq \frac{V_{d}-V_{c}}{d-c} x+\frac{d V_{c}-c V_{d}}{d-c} .
$$

4. Moreover, $V$ is the unique convex solution of $(1)$ in any of the above cases.

\section{Proof}

1. It is easily verified that $V(x)=\frac{V_{c}}{c} x$ satisfies the equation of (1) and $V(c)=V_{c}$, and that the boundary condition $V(d)=V_{d}$ results if and only if $\frac{V_{d}}{d}=\frac{V_{c}}{c}$.

2. Let $V_{*}$ denote a convex solution of the auxiliary problem (2) given by Theorem 1. Then, using (2) and Proposition 1, we have that

$$
\begin{aligned}
V_{*}^{\prime \prime}(x)=-H\left(x, V_{*}, V_{*}^{\prime}\right) & =-\frac{p x^{2}-\sqrt{p^{2} x^{4}+4 q x^{3}\left|x V_{*}^{\prime}-V_{*}\right|}}{2 x^{3}} \\
& =\frac{-p x^{2}+\sqrt{p^{2} x^{4}-4 q x^{3}\left(x V_{*}^{\prime}-V_{*}\right)}}{2 x^{3}} ;
\end{aligned}
$$

therefore $V_{*}$ is a solution of the equation

$$
x^{3}\left(V^{\prime \prime}\right)^{2}+p x^{2} V^{\prime \prime}+q\left(x V^{\prime}-V\right)=0 .
$$

Since $V_{*}(c)=V_{c}$ and $V_{*}(d)=V_{d}$, we conclude that $V_{*}$ is a solution of the Dirichlet boundary value problem (1). Statement (6) is obtained from Theorem 1.

3. The proof is the same as for the previous case.

4. The uniqueness result follows immediately from Theorem 2.1 of [3]. 


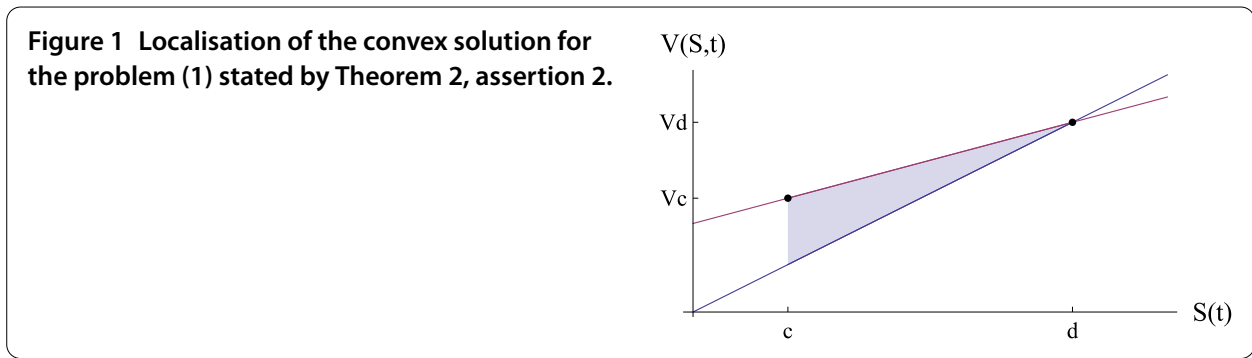

Figure 2 Localisation of the convex solution for the problem (1) stated by Corollary 1.

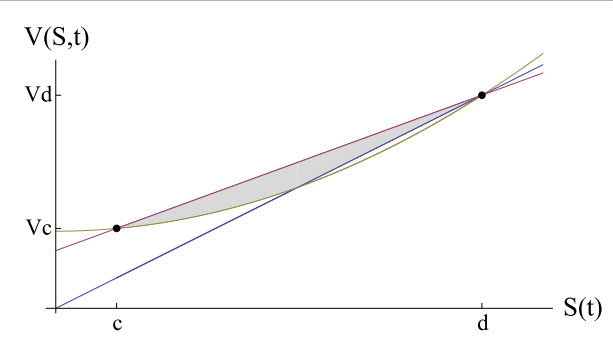

Remark 2 We remark that Theorem 2.1 of [3] guarantees the existence and uniqueness of a solution but does not give information on localisation, due to the method used. However, using that uniqueness result, from the above theorem, assertions 2 and 3 , we can derive the following corollary.

Corollary 1 Let $\frac{V_{d}}{d}<\frac{V_{c}}{c}$. If $k=\sqrt{\frac{p}{c^{3}}}$ is small enough, then the nonlinear Dirichlet boundary value problem (1) has a convex solution $V$ such that

$$
\max \left\{\frac{V_{d}}{d} x, \frac{V_{d}-V_{c}}{d^{2}-c^{2}} x^{2}+\frac{d^{2} V_{c}-c^{2} V_{d}}{d^{2}-c^{2}}\right\} \leq V(x) \leq \frac{V_{d}-V_{c}}{d-c} x+\frac{d V_{c}-c V_{d}}{d-c} .
$$

Figure 1 illustrates the localisation for $x \in[c, d]$ of the convex solution for the problem (1) whose existence is given by assertion 2 of Theorem 2 .

Assertion 3 of Theorem 2 improves the localisation result contained in assertion 2 as long as $k$ is small enough. In fact, more accuracy is obtained near $c$.

Figure 2 is an illustration of the new localisation of the convex solution in this case.

\section{Application to a generalised Black-Scholes model}

For better understanding how that above equation appears from financial option pricing, we present in this section some of the financial and mathematical features that lie behind the model. Namely, we will refer to the introduction of transaction costs in the classical Black-Scholes (BS) model. The classical BS model concerns the price of a call or a put option on an underlying asset when the price of this asset itself is modelled as a geometric Brownian motion. We consider the BS equation

$$
\frac{\partial V}{\partial t}+\frac{1}{2} \sigma^{2} S^{2} \frac{\partial^{2} V}{\partial S^{2}}+r S \frac{\partial V}{\partial S}-r V=0
$$

with the final condition, which represents the pay-off function,

$$
V(T ; S)=\phi(S)
$$


where $V$ is the option price, $S$ is the stock price at time $t, T$ is the maturity time, $r$ is the interest short rate and $\sigma$ is the volatility of the stock returns.

When approaching the question of introducing transaction costs in the classical BlackScholes model, one of the first main references is the work presented by Leland in [5], that suggests a market with proportional transaction costs. That is, considering $v$ the number of shares (it is positive if the agent buys or negative if the agent sells) and $S$ the price of the asset at time $t$, the costs of the transaction of $v$ shares at time $t$ are given by

$k S|v|$,

where the constant $k>0$ depends on the parts involved in the transaction.

Leland [5] suggested a new strategy using transaction costs proportional to the monetary value of any buy or sale of the asset. Leland's replication strategy consisted in using the common Black-Scholes formulae in periodical revisions of the portfolio but with an appropriately enlarged volatility. This is a model widely accepted in the financial industry. However, there are some mathematical problems with this approach, as referred by Kabanov and Safarian [6]: the terminal value of the replicating portfolio does not converge to the terminal payoff of the derivative if the transaction costs do not depend on the number of revisions (tending to infinity), limiting discrepancy that can be calculated explicitly.

In this work we use a slightly different structure for the transaction costs that was presented by Amster et al. [3]. We assume that the individual cost of the transaction of each share diminishes as the number of shares transactioned increases, which is represented by considering the cost as the percentage given by

$$
h(v)=a-b|v|,
$$

where $v$ is the number of shares traded ( $v>0$ in a buy or $v<0$ in a sale) and $a, b>0$ are constants depending on the individual investor.

We consider the hedging portfolio consisting in an option of value $V$ (short position) and $\Delta$ shares of the underlying asset of price $S$ (long position), and consider a strategy where the portfolio is reviewed every $\delta t$, with $\delta t$ a finite, fixed time step.

The inclusion of transaction costs in the classical Black-Scoles model leads us to the following equation: (see [4])

$$
\frac{\partial V}{\partial t}+\frac{1}{2} \sigma^{2} S^{2} \frac{\partial^{2} V}{\partial S^{2}}-a \sigma S^{2}\left|\frac{\partial^{2} V}{\partial S^{2}}\right| \sqrt{\frac{2}{\pi \delta t}}+b \sigma^{2} S^{3}\left(\frac{\partial^{2} V}{\partial S^{2}}\right)^{2}+r S \frac{\partial V}{\partial S}-r V=0,
$$

where $\mu$ is the drift coefficient and $\sigma$ is the volatility of the underlying asset.

Considering $a$ small enough such that

$$
\tilde{\sigma}^{2}=\sigma^{2}\left[1-\operatorname{sign}\left(\frac{\partial^{2} V}{\partial S^{2}}\right) \frac{2 a}{\sigma} \sqrt{\frac{2}{\pi \delta t}}\right]>0,
$$

the following nonlinear equation is obtained:

$$
\frac{\partial V}{\partial t}+\frac{1}{2} \tilde{\sigma}^{2} S^{2} \frac{\partial^{2} V}{\partial S^{2}}+b \sigma^{2} S^{3}\left(\frac{\partial^{2} V}{\partial S^{2}}\right)^{2}+r\left(\frac{\partial V}{\partial S} S-V\right)=0 .
$$


The equation obtained (10) is clearly an extension of the classical Black-Scholes equation. The introduction of our particular model for the transaction costs in the option pricing market led us to a partial differential equation that contains the Black-Scholes terms with an additional nonlinear term modelling the presence of transaction costs. We also use an adjusted volatility in the model $\tilde{\sigma}$, not the real volatility $\sigma$.

When concerned with the existence and localisation of stationary solutions of the above equation, in order to consider models where the influence of time does not play an important role, we are led to the following equation:

$$
b \sigma^{2} S^{3}\left(V^{\prime \prime}\right)^{2}+\frac{1}{2} \tilde{\sigma}^{2} S^{2} V^{\prime \prime}+r\left(V^{\prime} S-V\right)=0 .
$$

Dividing by the positive constant $b \sigma^{2}$ and writing $p=\frac{\tilde{\sigma}^{2}}{2 b \sigma^{2}}$ and $q=\frac{r}{b \sigma^{2}}$, we obtain

$$
S^{3}\left(V^{\prime \prime}\right)^{2}+p S^{2} V^{\prime \prime}+q\left(V^{\prime} S-V\right)=0 .
$$

This equation is precisely the equation of the problem (1) studied in the previous section. Contributions to better understanding of the analytical features of the model can be useful for future developments of the financial model itself. In fact, research is full of many examples that illustrate the fruitful interplay between finance and mathematics with reciprocal advantages.

\section{Competing interests}

The authors declare that they have no competing interests.

\section{Authors' contributions}

Both authors collaborated in all the steps concerning the research, discussion and achievements presented in the final manuscript.

\section{Author details}

${ }^{1}$ ISEG, CEMAPRE - Technical University of Lisbon, Rua do Quelhas 6, Lisboa, 1200-781, Portugal. ²Department of Mathematics, University of Trás-os-Montes e Alto Douro, Apartado 1013, Vila Real, 5001-801, Portugal.

\section{Acknowledgements}

This research was supported by the Fundação para a Ciência e Tecnologia through the project PEst-OE/EGE/UI0491/2011 and by the European Union in the FP7-PEOPLE-2012-ITN Program under Grant Agreement Number 304617 (FP7 Marie Curie Action, Project Multi-ITN STRIKE - Novel Methods in Computational Finance. We thank the anonymous referees for their useful comments.

Received: 29 December 2012 Accepted: 24 May 2013 Published: 13 June 2013

\section{References}

1. Mawhin, J: Points fixes, points critiques et problèmes aux limites. Séminaire de Mathématiques Supérieures, vol. 92. Presses University Montreal, Montreal (1985)

2. De Coster, C, Habets, P: Two-Point Boundary Value Problems Lower and Upper Solutions. Elsevier, Amsterdam (2006)

3. Amster, P, Averbuj, CG, Mariani, MC, Rial, D: A Black-Scholes option pricing model with transaction costs. J. Math. Anal. Appl. 303, 688-695 (2005)

4. Grossinho, MR, Morais, E: A note on a stationary problem for a Black-Scholes equation with transaction costs. Int. J. Pure Appl. Math. 51, 579-587 (2009)

5. Leland, HE: Option pricing and replication with transaction costs. J. Finance 40, 1283-1301 (1985)

6. Kabanov, Y, Safarian, M: Markets with Transaction Costs - Mathematical Theory. Springer Finance. Springer, Berlin (2009) 